\title{
Effectiveness and Risk Factors for Virological Outcome of Raltegravir-Based Therapy for Treatment-Experienced HIV-Infected Patients
}

\author{
José Antonio Mata-Marín ${ }^{1}$ - Ariane Estrella Weiser Smeke ${ }^{2}$ Mariana Rotzinger Rodriguez $^{2}$. \\ Marcelino Chávez-García ${ }^{3}$ - Marco Isaac Banda-Lara ${ }^{4}$ Alma Minerva Pérez Rios ${ }^{5}$. \\ Nohemí Nuñez-Rodríguez ${ }^{6} \cdot J^{\prime}$ Can Carlos Domínguez-Hermosillo ${ }^{1} \cdot$ Alberto Chaparro Sánchez $^{1}$ • \\ Irene Juarez-Kasusky ${ }^{7}$ Javier Enrique Cruz Herrera ${ }^{8} \cdot$ Jorge Luis Sandoval Ramírez $^{1}$. \\ Jesús Gaytán-Martínez ${ }^{1}$
}

Published online: 25 January 2017

(c) The Author(s) 2017. This article is published with open access at Springerlink.com

\begin{abstract}
Objective We evaluated the effectiveness of a raltegravir (RAL)-containing regimen plus an optimized background regimen in HIV-1 highly treatment-experienced patients. Design A retrospective cohort, multicentre study was conducted.

Methods Adult ( $>16$ years old) HIV treatment-experience patients starting therapy with a RAL-containing regimen were included. Effectiveness was evaluated as the percentage of patients with an undetectable HIV-1 RNA viral load $(<50$ and $<200$ copies $/ \mathrm{mL})$ after 48 weeks, and changes in CD4+ cell counts. We evaluated the risk factors associated with treatment failure.
\end{abstract}

Ariane Estrella Weiser Smeke

aweisers@gmail.com

1 Infectious Diseases Department, Hospital de Infectología, National Medical Center "La Raza”, IMSS, Mexico City, Mexico

2 Medicine School, Universidad Anáhuac, Campus norte, Av. Universidad Anáhuac 46, Lomas Anahuac, 52786

Naucalpan de Juárez, Mexico City, Mexico

3 Unidad Médica de Alta Especialidad No. 25, IMSS, Monterrey, Nuevo León, Mexico

4 CLISIDA, HGR San Luis Potosí, IMSS, SLP, Mexico City, Mexico

5 CLISIDA, HGR-110, IMSS, Guadalajara, Jalisco, Mexico

6 CLISIDA, HGZ-24, IMSS, Mexico City, Mexico

7 CLISIDA, HGZ-29, IMSS, Mexico City, Mexico

8 HGZ-72, Gustavo Baz, Tlalnepantla, Mexico City, Mexico
Results Of the 107 patients in the cohort, $86 \%$ were men, the median age was 45 years [interquartile range (IQR) 40-52] and the median number of previous regimens was six (IQR 4-7). After 48 weeks of treatment, 73\% (IQR 63-80\%) of patients $(n=78)$ had a viral load of $<50$ copies $/ \mathrm{mL}$ and $85 \%$ (IQR 77-90\%) $(n=91)$ had $<200$ copies/mL. In a logistic regression model, risk factors associated with a virological outcome of HIV-1 RNA of $<200$ copies $/ \mathrm{mL}$ were age $>40$ years [odds ratio (OR) 5.61; 95\% confidence interval (CI) 1.61-18.84; $P=0.006]$ and use of tenofovir in the regimen (OR 0.16; 95\% CI 0.03-0.80; $P=0.026$ ).

Conclusions In this Mexican cohort, RAL achieved high rates of virological suppression and an increase in CD4+ cell count in highly treatment-experienced patients infected with HIV-1. Age $>40$ years was associated with a good virological outcome, contrary to tenofovir use, which was associated with a poor virological outcome.

\section{Key Points}

Treatment with RAL has shown significant effectiveness in highly ARV-experienced HIVinfected patients, leading to an important virological and immunological response.

With regard to effectiveness, we found that the OBR played an important role in the achievement of the primary and secondary endpoints. Age $>40$ years was significantly associated with success, and TDF in the regimen was associated with failure leading to an HIV-1 RNA viral load of $<200$ copies $/ \mathrm{mL}$. 


\section{Introduction}

Antiretroviral therapy (ART) has changed the evolution of HIV infection, decreasing morbidity and mortality; however, it has some drawbacks [1]. Virological failure occurs in a significant proportion of patients [2] and represents one of the most challenging health management issues [3].

Raltegravir (RAL) is a first-strand-transfer inhibitor of HIV-1 integrase, an enzyme encoded by the HIV viral genome that catalyses the stepwise process of integration of the HIV-1 DNA into the genome of the host cell $[4,5]$. It is generally safe and well tolerated, with potent antiretroviral activity in both treatment-experienced and treatmentnaïve patients [6].

In the BENCHMRK (efficacy and safety of raltegravir for treatment of HIV for 5 years in the BENCHMRK studies: final results of two randomized, placebo-controlled trials) studies, RAL combined with an optimized background regimen (OBR) demonstrated superiority over OBR alone; these reports showed that HIV-1 RNA suppression and the increase in CD4+ cell count was better in patients taking RAL than in those taking placebo [7]. However, these trials have also shown that RAL has a low genetic barrier to resistance, which can rapidly emerge after virological failure and abolishes the antiviral activity of the drug [8].

RAL has a favourable profile related to interactions; thus it provides few constraints when designing treatment regimens. Its clinical pharmacology and drug-interaction profile make it well suited for diverse patient populations when it is incorporated into combination therapy with other antiretrovirals and supportive medications without dose modification [9].

The objective of the present study was to evaluate the virological and immunological effectiveness of RAL plus an OBR in a Mexican cohort of highly treatment-experienced patients infected with HIV-1.

\section{Methods}

\subsection{Design}

We performed a retrospective study on a cohort of 107 treatment-experienced HIV-1-infected adults who started therapy with a RAL-containing regimen in the period September 2009 to October 2013 who fulfilled the inclusion criteria. The first endpoint to be analysed was an HIV1 RNA viral load of $<50$ copies/mL after the patients had completed 48 weeks of treatment. The secondary endpoints were the proportions of patients with a HIV-1 RNA viral load of $<200$ copies/mL and changes in CD4+ cell count.
In addition, we also evaluated the safety and the risk factors associated with virological failure in experienced patients initiating RAL-based regimens.

\subsection{Ethics}

The study complies with current ethical principles. Data were abstracted from a database, so informed consent was not necessary.

\subsection{Patients}

Patients were recruited for HIV treatment from eight referral centres of five Mexican states. Patients included were $>16$ years old with confirmed HIV-1 infection, who had had experience with triple-class antiviral drugs and had mutations detected for at least two antiretroviral (ARV) classes, failing their current regimen. Those lost to followup or with treatment discontinuation secondary to other causes were also registered. Patients had been treated previously with at least three classes of ARV drugs including nucleoside analogue reverse-transcriptase inhibitors (NRTIs), non-nucleoside reverse-transcriptase inhibitors (NNRTIs), and protease inhibitors (PIs), but they were naïve to integrase-inhibitor drugs. An individualized OBR was chosen for each patient. The regimen included three to four ARV agents in order to achieve a genotype sensitivity score (GSS) of $\geq 2$ points based on HIV-1 resistance testing and previous ARV drug experience. All patients were on an antiretroviral regimen that exerted pressure before performing a genotype test. Once the results of each patient's genotype, tropism test and previous regimens were documented, an expert committee evaluated each case to decide the best option for a salvage regimen using RAL plus an OBR, considering the previous ARV regimens.

\subsection{Measurements}

Information extracted from clinical histories included ART regimens, CD4+ cell counts, HIV-1 RNA viral load, blood cell count, blood chemistry and lipids profile at the beginning of the therapy with RAL (baseline) and at 24 and 48 weeks later. Mutations were assessed from plasma HIV1 polymerase sequences using the Stanford HIV Drug Resistance Database (HIVdb; http://hivdb.stanford.edu). The presence of resistance was defined according to the Stanford HIVdb sensitivity score (SS) ranges as follows: 0-9 = susceptible; $10-14=$ potential low-level resistance; $15-29=$ low-level resistance; $30-59=$ intermediate resistance; and $\geq 60=$ high-level resistance. The GSS was defined as the total number of drugs (excluding RAL) in a participant's OBR ARV regimen to which their HIV 
isolate had genotypic sensitivity, as deduced from gene sequence and mutation analyses. Each ARV drug was assigned a score according to the five-level Stanford HIVdb interpretation. The sum of the individual scores for specific drugs provided the total GSS of that treatment, where $0-9=1,10-14=0.75,15-29=0.5,30-59=0.25$ and $\geq 60=0$. We classified the total GSS score in the following categories: $0-1,1-2$, or $\geq 2$. The $0-1$ group contains viral sequences almost entirely resistant to the drugs in the OBR regimen, and the $\geq 2$ group contains viral sequences susceptible to more than two drugs included in the regimen [9].

The effectiveness of RAL treatment was evaluated based on the percentages of patients with an undetectable HIV-1 RNA viral load after 48 weeks of treatment and the changes in CD4+ cell counts. We analysed the resistance-associated mutations (RAMs) associated with RAL at baseline, the OBR GSS and the Stanford HIVdb SS for potential risk factors of virological failure. Evaluations of metabolic safety were based on changes in fasting lipid levels [total cholesterol (TC) and triglycerides (TG)] and serum creatinine from baseline to week 48 .

\subsection{Statistical Analysis}

Baseline characteristics were summarized using medians and interquartile ranges (IQRs) for continuous variables, and proportions for categorical variables. To assess the effects of treatment, we calculated medians with IQRs for continuous variables and the number of values in each category and the percentages of the values with regard to the number of patients for categorical values. Nonparametric paired tests were used to evaluate changes in CD4+ cell counts and HIV-1 RNA viral load. Descriptive statistics were used to evaluate changes from baseline CD4+ cell counts and HIV-1 RNA viral load. Explorative statistical methods were used regarding the efficacy endpoints and changes in safety-relevant laboratory parameters. The significance of changes from baseline was tested using the Wilcoxon signed-rank test. We calculated the $95 \%$ confidence interval (CI) for appropriate results.

Baseline differences between patients who reached or did not reach a viral load of $<50$ copies $/ \mathrm{mL}$ at week 48 were tested using bivariate analysis, which included crude odds ratios (ORs), Fisher's exact and chi-squared tests. Independent risk factors associated with virological response at week 48 were identified in a multivariate logistic regression analysis that included variables from the bivariate analysis. All analyses were performed using SPSS software (IBM SPSS Statistics for Windows, version 19.0; IBM Corp., Armonk, NY, USA).
Table 1 Baseline patient characteristics and composition of OBR

\begin{tabular}{ll}
\hline Characteristics & Values \\
\hline Age, years & $45(40-52)$ \\
Male, $n(\%)$ & $92(86)$ \\
Number of previous regimens & $6(4-7)$ \\
Years of treatment experience & $12(9-17)$ \\
Baseline HIV-1 plasma viral load, & $23,100(4512-75,772)$ \\
copies/mL & \\
Baseline HIV-1 RNA $>100,000$ copies/mL, & $20(18.7)$ \\
$n(\%)$ & \\
Baseline CD4+ cell counts (cells/ $\mu \mathrm{L})$ & $244(128-404)$ \\
Baseline CD4+ cells count $<200$ cells/ $\mu \mathrm{L}$, & $40(37.4)$ \\
$n(\%)$ & \\
GSS for OBR & $1.25(1.0-2.0)$ \\
DRV in regimen, $n(\%)$ & $102(95)$ \\
TDF in regimen, $n(\%)$ & $66(61.7)$ \\
ETV in regimen, $n(\%)$ & $28(26.2)$ \\
ENF in regimen, $n(\%)$ & $13(12.1)$ \\
EFV in regimen, $n(\%)$ & $8(7.5)$ \\
MVC in regimen, $n(\%)$ & $8(7.5)$ \\
Stanford score for TDF & $55(25-65)$ \\
Stanford score for ETV & $0(0-30)$ \\
Stanford score for DRV & $15(0-20)$ \\
Number of PI RAMs, for DRV & $1(1-2)$ \\
Number of PI RAMs, non-DRV RAMs & $4(3-6)$ \\
Glucose (mg/dL) & $85(78-91)$ \\
Creatinine (mg/dL) & $0.85(0.70-1.0)$ \\
ALT (IU/dL) & $30(19-48)$ \\
Total cholesterol (mg/dL) & $166(137-200)$ \\
Triglycerides (mg/dL) & $193(137-264)$ \\
& \\
&
\end{tabular}

Values are $n(\%)$ or median (IQR)

$A L T$ alanine aminotransferase, $D R V$ darunavir, GSS genotypic sensitivity score, $E F V$ efavirenz, $E N F$ enfuvirtide, $E T V$ etravirine, $I Q R$ interquartile range, $M V C$ maraviroc, $O B R$ optimized background regimen, $P I$ protease inhibitor, $R A M$ resistance-associated mutation, $T D F$ tenofovir

\section{Results}

One hundred and seventeen multidrug-experienced patients who initiated a RAL-based salvage therapy between 2009 and 2013 were identified. Only 107 patients who had complete data and had been followed through 48 weeks were included. The median age at RAL initiation was 45 years (IQR 40-52), 86\% were men, and 87 (81\%) were older than 40 years of age. US Centers of Disease Control and Prevention (CDC) class C AIDS was found in $68 \%$ of patients, and the median number of previous ARV treatments was six (IQR 4-7). The median GSS of the OBR for all patients was 1.25 (IQR 1.0-2.0). Twenty-nine patients (27\%) had a GSS of $\geq 2$ (Table 1 ). 
Table 2 Bivariate analysis of factors associated with an outcome of HIV-1 RNA $<50$ copies/mL at week 48 of antiretroviral treatment

\begin{tabular}{llll}
\hline Risk factor & \multicolumn{2}{l}{ Bivariate } & \multirow{2}{l}{$P$ value } \\
\cline { 2 - 3 } & OR unadjusted & $95 \% \mathrm{CI}$ & \\
\hline Male sex & 1.02 & $0.29-3.52$ & 0.967 \\
Age $>40$ years & 2.09 & $0.77-5.81$ & 0.150 \\
Number of previous regimens $>6$ & 0.839 & $0.28-2.44$ & 0.746 \\
Baseline HIV-1 RNA $>100,000$ copies $/ \mathrm{mL}$ & 0.47 & $0.17-1.32$ & 0.150 \\
Baseline CD4+ cell count $<200$ cells $/ \mu \mathrm{L}$ & 0.44 & $0.18-1.00$ & 0.050 \\
GSS $\geq 2$ & 1.22 & $0.48-3.10$ & 0.674 \\
TDF & 0.64 & $0.26-1.60$ & 0.34 \\
ETV & 1.15 & $0.43-3.10$ & 0.77 \\
EFV & 1.11 & $1.03-1.20$ & 0.073 \\
MVC & 1.11 & $1.03-1.20$ & 0.073 \\
ENF & 0.37 & $0.11-1.23$ & 0.09 \\
\hline
\end{tabular}

$C I$ confidence interval, $E F V$ efavirenz, ENF enfuvirtide, ETV etravirine, GSS genotypic sensitivity score, $M V C$ maraviroc, $O R$ odds ratio, $T D F$ tenofovir

\begin{tabular}{llll}
\hline Risk factor & \multicolumn{2}{l}{ Bivariate } & $P$ value \\
\cline { 2 - 3 } & OR unadjusted & $95 \%$ CI & \\
\hline Male sex & 0.413 & $0.113-1.506$ & 0.234 \\
Age $>40$ years & 4.66 & $1.47-14.72$ & 0.005 \\
Number of previous regimens $>6$ & 0.64 & $0.18-2.24$ & 0.490 \\
Baseline HIV-1 RNA $>100,000$ copies $/ \mathrm{mL}$ & 0.43 & $0.13-1.43$ & 0.171 \\
Baseline CD4+ cell count $<200$ cells $/ \mu \mathrm{L}$ & 0.40 & $0.13-1.18$ & 0.091 \\
GSS $\geq 2$ & 1.67 & $0.55-5.10$ & 0.361 \\
TDF & 0.19 & $0.04-0.88$ & 0.025 \\
ETV & 2.80 & $0.59-13.19$ & 0.229 \\
EFV & 1.09 & $1.02-1.16$ & 0.603 \\
MVC & 1.09 & $1.02-1.16$ & 0.603 \\
ENF & 0.535 & $0.13-2.20$ & 0.408 \\
\hline
\end{tabular}

$C I$ confidence interval, $E F V$ efavirenz, ENF enfuvirtide, ETV etravirine, GSS genotypic sensitivity score, $M V C$ maraviroc, $O R$ odds ratio, $T D F$ tenofovir
Table 3 Bivariate analysis of risk factors associated with a virological outcome of HIV-1 RNA $<200$ copies/mL at week 48 of antiretroviral treatment
Darunavir (DRV) was used in 102 patients $(95 \%)$, three patients $(2.8 \%)$ used a tipranavir-containing regimen, and two patients $(1.8 \%)$ used a PI-free regimen.

The most frequent RAMs for DRV were I84V (35.8\%), L33F (34.9\%), L10F (19.8\%), I47V (10.4\%) and V32I (7.5\%).

At baseline, the median HIV-1-RNA viral load was 23,100 copies/mL $\left(4.36 \log _{10}\right)$ with an IQR of 4512-75,772 copies/mL (3.6-4.8 $\left.\log _{10}\right)$.

After 48 weeks of treatment, 73\% (IQR 63-80\%) of patients $(n=78)$ had an HIV-1 RNA viral load $<50$ copies/mL and $85 \%$ (IQR 77-90\%) $(n=91)$ had $<200$ copies/mL. Twenty-nine patients $(27 \%)$ had treatment failure; of them, ten $(9.3 \%)$ fulfilled virological failure criteria, two $(1.8 \%)$ were lost to follow-up and four $(3.7 \%)$ discontinued treatment because of toxicity related to the regimen (Tables 2, 3).
A logistic regression model showed that the risk factors associated with virological outcome in HIV-1 RNA $<200$ copies $/ \mathrm{mL}$ were age $>40$ years [OR $5.61(95 \% \mathrm{CI}$ 1.61-18.84); $P=0.006]$ and use of tenofovir (TDF) in the regimen [OR 0.16 (95\% CI 0.03-0.80); $P=0.026$ ].

At baseline, the median and IQR values for the CD4+ cell counts were 244 cells $/ \mu \mathrm{L}(128-404$ cells $/ \mu \mathrm{L})$, whereas at week 24 and 48 the values were 310 cells/ $\mu \mathrm{L}$ (239-523 cells/ $\mu \mathrm{L})$ and 383 cells $/ \mu \mathrm{L}(258-564$ cells $/ \mu \mathrm{L})$, respectively. Both these increases were significant $(P<0.001)$.

For assessing metabolic safety, fasting lipid profiles of TC and TG were measured, and are shown below as medians and IQRs. TC showed a significant increase $(P=0.003)$ from a baseline of $166 \mathrm{mg} / \mathrm{dL}(137-200 \mathrm{mg} /$ $\mathrm{dL})$ to $179 \mathrm{mg} / \mathrm{dL}(161-214 \mathrm{mg} / \mathrm{dL})$ at week 24, and it remained significantly elevated at $191 \mathrm{mg} / \mathrm{dL}$ 
Table 4 Endpoints after 24 and 48 weeks of treatment

\begin{tabular}{lllll}
\hline Outcomes & \multicolumn{2}{l}{ Median (IQR) } & & $\begin{array}{l}P \text { value } \\
\text { nyln }\end{array}$ \\
\cline { 2 - 4 } & Baseline & Week 24 & Week 48 & 24/48 weeks \\
\hline CD4+ cell count & $244(128-404)$ & $310(239-523)$ & $383(258-564)$ & $<0.001 /<0.001$ \\
HIV-1 RNA viral load & $23,100(4512-75,772)$ & $<20(<20-76)$ & $<20(<20-69)$ & $<0.001 /<0.001$ \\
Cholesterol (mg/dL) & $166(137-200)$ & $179(161-214)$ & $197(157-213)$ & $0.001 /<0.001$ \\
Triglycerides (mg/dL) & $193(137-264)$ & $193(156-271)$ & $216(169-303)$ & $0.63 / 0.085$ \\
Creatinine (mg/dL) & $0.85(0.70-1.00)$ & $0.90(0.80-1.1)$ & $0.90(0.80-1.1)$ & $0.001 / 0.001$ \\
\hline
\end{tabular}

$I Q R$ interquartile range
(157-213 mg/dL; $P<0.001)$ at 48 weeks. TG showed no significant increase from a baseline of $193 \mathrm{mg} / \mathrm{dL}$ (137-264 mg/dL), increasing to $193 \mathrm{mg} / \mathrm{dL}$ (156-271 mg/ $\mathrm{dL})$ at week 24 and to $216 \mathrm{mg} / \mathrm{dL}(169-303 \mathrm{mg} / \mathrm{dL})$ at week 48. Creatinine also showed a significant increase $(P=0.001)$ from a baseline of $0.85 \mathrm{mg} / \mathrm{dL}(0.7-1.0 \mathrm{mg} /$ $\mathrm{dL})$ to $0.9 \mathrm{mg} / \mathrm{dL}(0.8-1.1 \mathrm{mg} / \mathrm{dL} ; P=0.001)$ at week 24 , and remained high at week $48[0.9 \mathrm{mg} / \mathrm{dL}(0.8-1.1 \mathrm{mg} / \mathrm{dL}$; $P=0.001)]$. Increase in creatinine was higher in patients with a TDF-containing regimen $[0.9 \mathrm{mg} / \mathrm{dL}(0.8-1.0 \mathrm{mg} /$ dL) vs. $0.8 \mathrm{mg} / \mathrm{dL}(0.9-1.1 \mathrm{mg} / \mathrm{dL})]$ than in the group without $\operatorname{TDF}(P=0.04)$ (Table 4$)$.

\section{Discussion}

In this study, treatment with RAL has shown significant effectiveness in highly ARV-experienced HIV-infected patients, leading to an important virological and immunological response.

With regard to effectiveness, we found that the OBR played an important role in the achievement of the primary and secondary endpoints. Age $>40$ years was significantly associated with success, and TDF in the regimen was associated with failure leading to an HIV-1 RNA viral load of $<200$ copies/mL.

We observed that $73 \%$ of patients achieved a viral load of $<50$ copies $/ \mathrm{mL}$ at week 48 . This outcome was better than those of several other randomized clinical trials [5]. However, it was similar to the results obtained in the REALMRK (safety, tolerability, and efficacy of raltegravir in a diverse cohort of HIV-infected patients: 48-week results from the REALMRK study) and BENCHMRK trials, which evaluated different populations. REALMRK included mostly women and African-Americans in the USA, but also people from other countries, including Brazil, Dominican Republic, Jamaica and South Africa. BENCHMRK-1 included populations from Europe, Asia/ Pacific and Peru, and BENCHMRK-2 included populations from North and South America. In the combined RAL groups from both studies, $57 \%$ of patients at week 96 and
$51 \%$ at week 156 had viral loads of $<50$ copies $/ \mathrm{mL}$ $(P<0.0001)$. CD4+ cell counts increased on average from 125 cells $/ \mu \mathrm{L}$ at week 96 to 164 cells $/ \mu \mathrm{L}$ at week 156 in the RAL group, and from 49 to 63 cells $/ \mu \mathrm{L}$ in the placebo group [6].

In the SALIR-E (four years data of raltegravir-based salvage therapy in HIV-1-infected, treatment-experienced patients: the SALIR-E study), 73\% were HIV-1 RNA undetectable at week 206; in a multivariate analysis of predictors of interruption or failure, each baseline HIV-1 RNA $\log _{10}$ increase was associated with an adjusted hazard ratio for failure of 1:6; having more than 13 previous treatments also emerged as a predictor; similar with our study, $73 \%$ of patients were HIV-1 RNA undetectable, but the predictors for treatment failure are different [10].

In the Antiviral Response Cohort Analysis (ARCA) database, virological response was achieved in $74.3 \%$ of 105 subjects, similar to our data [11]. In contrast, GSS had borderline statistical significance. When stratifying at different cut-offs ( $<1$ as reference, $1-1.49, \geq 1.5$ ), a borderline significant increase in the probability of response appeared for a GSS of $\geq 1.5$. A GSS of $\geq 1$ showed the highest sensitivity, $82.6 \%$. When we analysed according to GSS cut-offs, we found an association with virological response. The INI-VAIN (virological failure to raltegravir in Spain: incidence, prevalence and clinical consequences) study group evaluated the incidence, prevalence and clinical consequences of virological failure of RAL-based regimens in Spain [12]. In this study, 106 patients (55\%) that started a RAL-based regimen as part of a salvage ART treatment demonstrated in the multivariate analysis that the independent predictors for virological failure were plasma viral load at initiation of salvage ART and estimated adherence, $90 \%$ according to clinical records. In our study, independent predictors for virological failure are different, and we were not able to evaluate adherence.

In previous clinical studies, the concurrent use of DRV and RAL has been associated with a favourable treatment outcome. Some trials have found a synergistic effect, including the BENCHMRK trial, where the co-administration of RAL and DRV/ritonavir (DRV/r) improved the 
outcome [7]. In that trial, HIV-1 RNA levels of $<50$ copies/ $\mathrm{mL}$ were achieved at 48 weeks in $47 \%$ of recipients of DRV/r plus an OBR, compared with $69 \%$ of patients who received RAL and DRV/r plus an OBR.

In our study, eight patients treated with maraviroc and efavirenz reached $<50$ copies $/ \mathrm{mL}$ at 48 weeks of treatment; however, this result was not statistically significant. In contrast, an OBR that included TDF was found to increase the risk of virological failure. We found that intermediate resistance for TDF was associated with virological failure; there were few options of ARV available in Mexico when TDF was used, and although TDF had compromised activity, it was an option. Therefore, TDF was used in salvage regimens even though it had low activity. That is why we consider this to be a factor associated with virological failure in the population we studied. We did not find an association of DRV with PI mutations; I84V was the most frequent mutation in our patients with intermediate- or high-level resistance, and this was associated with an increased risk of virological failure in other studies [6].

Regarding metabolic safety, rescue therapy was associated with significantly greater rises in TC and serum creatinine. TG did not vary significantly. As seen in the SPIRAL study [13] and in other randomized clinical trials, RAL usually results in a better lipid profile than other ARTs. We found that increase of creatinine was higher in patients with a TDF-containing regimen.

This is the first multicentre cohort study in Mexico that has evaluated the effectiveness of RAL in highly treatmentexperienced patients. The relevance of this study is that we were able to evaluate risk factors for virological failure in our population. However, the study has limitations because it has a small sample size and is a retrospective study with previously enrolled patients in which we were not able to establish adverse events associated when starting the regimen, nor could we assess patient compliance for ARV drugs.

Despite these limitations, the results of the current analyses suggest that RAL is an effective treatment for highly ARV-experienced HIV-1 infected patients; therefore, our results provide a good starting point from which to establish an evidence base for management of virological failure in Mexican patients.

Acknowledgements The authors wish to thank all the centres and investigators who participated in this study.

\section{Compliance with Ethical Standards}

Conflict of Interest and Sources of Funding The content is solely the responsibility of the authors and does not necessarily represent the official views of the Mexican Institute of Social Security. JAMM has served as an advisor and received honoraria for medical education from Merck Sharp \& Dohme (MSD), Janssen and Bristol. MCG has served as an advisor and received honoraria for medical education from MSD, Janssen, Roche and Abbvie. JGM has served as an advisor and received honoraria for medical education from MSD, Janssen, Roche and Abbvie. AMPR has served as an advisor and received honoraria for medical education from MSD, Roche, BMS, Abbvie, Pfizer, Gilead, Janssen, Glaxo and Novartis. AEWS, MRR, JCDH, MIBL, NNR, JECH, JLSR, IJK and ACS declare that they have no competing interest. The study complies with current ethical considerations; informed consent was not necessary.

Funding The project was supported by the authors.

Open Access This article is distributed under the terms of the Creative Commons Attribution-NonCommercial 4.0 International License (http://creativecommons.org/licenses/by-nc/4.0/), which permits any noncommercial use, distribution, and reproduction in any medium, provided you give appropriate credit to the original author(s) and the source, provide a link to the Creative Commons license, and indicate if changes were made.

\section{References}

1. Wolff M, Shepherd BE, Cortés C, et al. Clinical and virologic outcomes after changes in first antiretroviral regimen at 7 sites in the Caribbean, Central and South America Network. J Acquir Immune Defic Syndr. 2016;71:102-10.

2. Mocroft A, Ledergerber B, Viard JP, et al. Time to virological failure of 3 classes of antiretrovirals after initiation of highly active antiretroviral therapy: results from the EuroSIDA study group. J Infect Dis. 2004;190:1947-56.

3. Mata-Marín JA, Huerta-García G, Domínguez-Hermosillo JC, et al. Effectiveness and risk factors for virological outcome of darunavir-based therapy for treatment-experienced HIV-infected patients. AIDS Res Ther. 2015;12:31.

4. Cocohoba J, Dong BJ. Raltegravir: the first HIV integrase inhibitor. Clin Ther. 2008;30:1747-65.

5. Eron JJ, Rodgers AJ, Cooper DA, et al. Association between first year virological response to raltegravir and long-term outcomes in treatment-experienced patients with HIV-1 infection. Antivir Ther. 2015;20:307-15.

6. Squires KE, Bekker LG, Eron JJ, et al. Safety, tolerability, and efficacy of raltegravir in a diverse cohort of HIV-infected patients: 48-week results from the REALMRK study. AIDS Res Hum Retrovir. 2013;29:859-70.

7. Eron JJ, Cooper DA, Steigbigel RT, et al.; BENCHMRK Study Teams. Efficacy and safety of raltegravir for treatment of HIV for 5 years in the BENCHMRK studies: final results of two randomised, placebo-controlled trials. Lancet Infect Dis. 2013;13:587-96.

8. Gallien S, Braun J, Delaugerre C, et al.; EASIER ANRS 138 Study Group. Efficacy and safety of raltegravir in treatment-experienced HIV-1-infected patients switching from enfuvirtidebased regimens: 48 week results of the randomized EASIER ANRS 138 trial. J Antimicrob Chemother. 2011;66:2099-106.

9. Brainard DM, Wenning LA, Stone JA, Wagner JA, Iwamoto M. Clinical pharmacology profile of raltegravir, an HIV-1 integrase strand transfer inhibitor. J Clin Pharmacol. 2011;51:1376-402.

10. Capetti A, Meraviglia P, Landonio S, et al. Four years data of raltegravir-based salvage therapy in HIV-1-infected, treatmentexperienced patients: the SALIR-E study. Int J Antimicrob Agents. 2014;43:189-94.

11. Rusconi S, Vitiello P, Adorni F, et al. Factors associated with virological success with raltegravir-containing regimens and 
prevalence of raltegravir-resistance-associated mutations at failure in the ARCA database. Clin Microbiol Infect. 2013;19:936-42.

12. Santos JR, Blanco JL, Masiá M, et al. Virological failure to raltegravir in Spain: incidence, prevalence and clinical consequences. J Antimicrob Chemother. 2015;70:3087-95.
13. Curran A, Martinez E, Saumoy M, et al. Body composition changes after switching from protease inhibitors to raltegravir: SPIRAL-LIP substudy. AIDS. 2012;26:475-81. 